\title{
Etiology and familial inheritance of pleomorphic adenomas
} Krysten Clark $^{1}$

${ }^{1}$ University of Pittsburgh, School of Dental Medicine

\section{Abstract}

Background: A pleomorphic adenoma is the most common salivary gland neoplasm in both children and adults. Pleomorphic adenomas are derived from ductal and myoepithelial cells and are most commonly found in the superficial lobe of the parotid gland. The purpose of this article is to discuss the genes involved in pleomorphic adenomas and the possible autosomal dominant mode of inheritance. Case Description: The first patient was a white male who was diagnosed with carcinoma ex pleomorphic adenoma, a highly aggressive tumor, at the age of 57 . He had an undiagnosed pleomorphic adenoma for approximately 15 years prior. The tumor was excised and the patient underwent radiation in the location of his parotid gland for 4 years until he deceased. The second patient is a white female, his daughter, who was diagnosed with a benign pleomorphic adenoma at the age of 46 . Her salivary gland tumor was excised and normal follow up appointments occurred. Practical Implications: Pleomorphic adenomas most commonly affect the parotid gland, the largest of the three major salivary gland tumors. Occurrence and excision of this salivary gland tumor will cause a decrease in the secretion of saliva, leading to a dry mouth and an increased risk of caries.
Citation: Clark, K. (2017) Etiology and familial inheritance of pleomorphic adenomas. Dentistry 3000. 1:a001 doi:10.5195/d3000.2017.70

Received: May 9, 2017

Accepted: May 10, 2017

Published: June 16, 2017

Copyright: (C2017 Clark, K. This is an open access article licensed under a Creative Commons Attribution Work 4.0 United States License.

Email: kdc40@pitt.edu

\section{Introduction}

Pleomorphic adenomas represent about $65 \%$ of all salivary gland neoplasms. They are the most common salivary gland neoplasm in both children and adults. The majority occur in the parotid gland, although it is possible for them to arise in minor salivary glands or the submandibular and sublingual glands. It is a painless, slow growing mass that can be palpated just behind the ear once large enough. They are defined as mixed tumors because they contain both epithelial, mesenchymal, and myoepithelial cells. Histologically, pleomorphic adenomas are arranged in various patterns between these three types of cells [1].

Pleomorphic adenomas most commonly arise in 4th decade of life and have a slight predilection towards females. The prognosis of a pleomorphic adenoma is typically very good, and the chance of malignancy is less than $5 \%$ if it is diagnosed within a few years. This chance increases up to $9.5 \%$ after 15 years [1]. The rate of recurrence is under $5 \%$ as long as complete excision occurs. However, if the tumor is poorly encapsulated, the rate of recurrence can be quite high. The treatment for a pleomorphic adenoma consists of removal of the tumor and surrounding salivary gland tissue, leading to a decreased salivary flow.

\section{Carcinoma ex pleomorphic} adenoma is a rare and aggressive malignant tumor that can arise from a pleomorphic adenoma. It represents only about $12 \%$ of malignant salivary gland tumors [1]. Normally, the risk of malignancy is very low unless the initial neoplasm is untreated for a prolonged period of time or recurrence of the primary tumor occurs. The mass is usually painless but can cause facial nerve paralysis. Treatment includes complete excision of the tumor, most likely followed by radiation. During the time of radiation, it is extremely important for the patient to visit a dentist. The five-year survival rate of carcinoma ex pleomorphic adenoma is only about $30 \%$. The purpose of this report is to to provide evidence for an autosomal dominant mode of inheritance of pleomorphic adenomas.

Subjects

A 46-year-old female patient was diagnosed with a pleomorphic adenoma of the parotid gland by an ENT after her dentist palpated a lump in the area during a routine head and neck exam. The tumor was excised, defined as benign, and after follow up appointments for 5 years, no further treatment was necessary. The likelihood of recurrence in her case is under 5\% [2].. This patient's father was diagnosed with carcinoma ex pleomorphic adenoma at the age of 57, and deceased 4 years later after metastatic spread to his lungs. This patient also had the tumor excised and received radiation. Upon diagnosis of (c) BY

ULIS D-Sente
New articles in this journal are licensed under a Creative Commons Attribution 4.0 United States License.

This journal is published by the University Library System, University of Pittsburgh as part of its D-Scribe Digital Publishing Program and is cosponored by the University of Pittsburgh Press. 


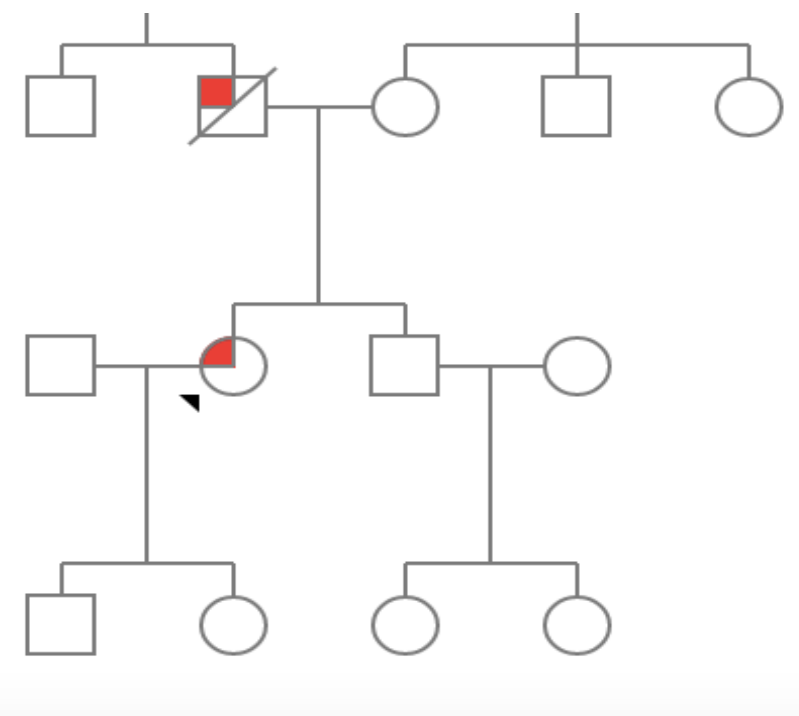

Figure 1: Family pedigree. Patient 1 is in generation 1 and had carcinoma ex pleomorphic adenoma after approx. 15 years without removal of primary tumor. Patient 2 is in generation 2 and had a pleomorphic adenoma. Offspring in generation 3 are still in their 20's and younger than the average age range for diagnosis.

his malignant tumor, he admitted to feeling a lump in the same location for at least 15 years. Figure 1 suggests a family segregation compatible with autosomal dominant mode of inheritance.

\section{Discussion}

The exact cause of pleomorphic adenomas is unknown, although $70 \%$ of cases show some form of chromosomal abnormality. There are both genetic and environmental risk factors. One well known environmental risk factor is exposure to therapeutic radiation. According to research, patients treated with radiation for childhood malignancies had an increased risk of developing a salivary gland neoplasm. Survivors of the atomic bomb drop have also shown an increased risk. However, many of these developing tumors were malignant mucoepidermoid carcinomas instead of pleomorphic adenomas [2]. Also, according to research studies, smoking surprisingly does not seem to have a strong association with the development of pleomorphic adenomas, although it plays a significant role in the development of Warthin's tumor, a different type of benign salivary gland tumor that also commonly affects the parotid gland [3]. The onset and progression of pleomorphic adenomas has also potentially thought to be linked to the use of mobile phones, although more research needs to be done. One last environmental risk factor to the formation of pleomorphic adenomas has viral origin. Although EBV and cytomegalovirus do not seem to be correlated, infection with the Simian virus, a highly oncogenic tumor virus, seems to play a role in the development of pleomorphic adenomas [4].

The simian virus (SV40) was originally in monkeys, but accidentally transferred to many humans who received an anti-polio vaccine. However, based on research, it seems that even individuals who did not receive the polio vaccine have been infected with SV40[4]. The virus has recently been found to be involved with different types of human tumors, including brain and bone tumors, mesotheliomas, thyroid carcinomas, and pituitary adenomas. Through the use of PCR, it was discovered that the SV40 Tag sequence was often found in pleomorphic adenomas but not in normal salivary gland tissue [4]. These results indicate that the Simian virus likely plays a role in the onset or progression of pleomorphic adenomas.

Three genes have been correlated with the development of pleomorphic adenomas. The first is pleo- morphic adenoma gene1 (PLAG1), which is found on chromosome 8 and present in about $39 \%$ of diagnosed patients [5]. The second gene is the high mobility group AT-hook 2 gene (HMGA2), located on chromosome 12 and found in $8 \%$ of patients. The above two mutations are both translocations. Lastly, the mucin 1 gene (MUC1) has been correlated to the recurrence and malignant transformation of pleomorphic adenomas.

PLAG1 and HMGA2 mutations are both proto-oncogenes, also known as gain of function mutations. Proto-oncogenes are normal genes in the body that are converted to oncogenes by three different mechanisms. The first mechanism is a point mutation that results in a continuously activated protein. The second mechanism is localized duplication of a DNA segment, leading to gene amplification and overexpression of that protein. The third mechanism is chromosomal translocation that causes a different promoter to promote inappropriate expression of a protein that is normally under gene control [6]. HMGA2 and most commonly PLAG1 are both transformed into oncogenes by translocation. However, trisomy 8 is a possible mechanism for the overexpression of PLAG1.

\section{PLAG1, found on chromo-} some 8 , has been consistently rearranged and over-expressed in individuals with pleomorphic adenomas. The most common PLAG1 translocation is found at $8 \mathrm{q} 12$, leading PLAG1 to be ubiquitously expressed. The two most common translocation genes are CTNNB1 found on chromosome 3 and LIFR found on chromosome 5 [5]. Also, mosaic trisomy 8 is the most common chromosomal deviation associated with salivary gland tumors [7]. Based on recent research it has been noted that PLAG1 acts as a pro- 
to-oncogene, and the overexpression of this gene is crucial to the development of pleomorphic adenomas. It has also been noted that the role of PLAG1 is confined to pleomorphic adenomas and carcinoma ex pleomorphic adenoma and does not play a role in other types of salivary gland neoplasms. PLAG1 encodes a zinc finger protein and activates DNA transcription by binding to DNA in a sequence-specific manner. One potential binding site of PLAG1 was found on the promoter region of human insulin-like growth factor II (IGF-II), leading to its overexpression [7]. The activation of IGFII causes growth promoting activity, which could lead to the formation of a tumor.

Mutations in HMGA2 seem to be more common in carcinoma ex pleomorphic adenoma, and are only found in around $8 \%$ of benign pleomorphic adenomas. HMGA2, a small non-histone associated protein located on chromosome 12 , can modify transcription by altering the chromatin structure. This gene also mutates through translocation, leading to the expression of $H M G A 2$, loss of regulation, and promotion of cell growth [8]. The amplification of this gene has been suggested to play a role in the malignant transformation of pleomorphic adenomas. Since both PLAG1 and $H M G A 2$ mutations are consistent with pleomorphic adenoma, the detection of these mutations can aid in the diagnosis.

Although the recurrence rate of pleomorphic adenomas is low in many cases where surgical excision is properly performed, if recurrence does occur, the chance of rerecurrence is high. These tumors are often multi-nodular and poorly encapsulated. Mucin-1 gene is one of the glycoproteins that makes up mucus. There is no expression of MUC1 in normal salivary glands. However, in a study by Hamada et al, the MUC1/DF3 mutation was found in 4 of 9 patients with recurrent pleomorphic adenomas, but only in 3 of 40 patients with non-recurrent pleomorphic adenomas [9]. According to literature, the expression of MUC1/DF3 could be used as a marker to detect the possible recurrence and malignant transformation of pleomorphic adenomas.

The evidence shows that pleomorphic adenomas do tend to have a familial component and the two modes of inheritance for pleomorphic adenomas are autosomal dominant inheritance and a somatic mutation [7]. If pleomorphic adenomas are passed in an autosomal fashion, a patient's offspring would have a $50 \%$ chance of passing the disease down to offspring. Based on the presented case, the first male patient likely had a mutation and passed the mutated gene to his daughter in an autosomal dominant type fashion. However, further research needs to be done to potentially link familial inheritance to the PLAG1 mutation, the mutated gene most commonly found in pleomorphic adenomas.

Pleomorphic adenomas can cause an increase in dental caries. The parotid gland is entirely serous and drains into the oral cavity through Stenson's duct. It produces approximately $20 \%$ of the salivary content in the oral cavity. The submandibular gland, produces around $65 \%$ of saliva and enters the oral cavity through Wharton's duct. Although pleomorphic adenomas most typically arise in the parotid gland, it can arise in either of the major salivary glands. Complete removal of these tumors causes a decrease in the production of one of the major salivary glands. This will almost certainly lead to a decrease in the production of saliva. Saliva plays an essential role in the prevention of caries, and a decreased salivary flow in the oral cavity makes an individual more prone to the development of caries. If a pleomorphic adenoma does develop into carcinoma ex pleomorphic adenoma and radiation therapy is required after surgical removal, the likelihood of caries increases exponentially. Radiation therapy causes an individual to lose almost all salivary flow, and frequent dental visits are required during this time.

Genetic testing for pleomorphic adenomas could cause potentially lead to ethical implications related to insurance companies. Also, there may be a fine line between normal and abnormal palpation during a head and neck exam. It is very important that a dentist refers a patient to a specialist if they feel anything abnormal, and missing a mass in a major or minor salivary gland could be an ethical implication. Overall, pleomorphic adenomas are very treatable when caught early and do not recur. There does seem to be a genetic link between pleomorphic adenomas and a few genes, like the $P L A G 1$ gene. More research needs to be completed on families with history of pleomorphic adenoma to discover a possible autosomal mode of inheritance.

\section{References}

1. PLAG1 gene alterations in salivary gland pleomorphic adenoma and carcinoma ex-pleomorphic adenoma: a combined study using chromosome banding, in situhybridization and immunocytochemistry. Martins C, Fonseca I, Roque L, Pereira T, Ribeira C, Bullerdiek J, 
Soares J. Modern Pathology. 2005; 18:1048-1055. 6 May 2006. PMID: 15920557.

2. Salivary gland tumors in survivors of childhood cancer. Whatley WS, Thompson JW, Rao B. 2006 March; 124 (3):385-388. PMID: 16500432

\section{Smoking and risk of parotid} gland tumors: a nationwide casecontrol study. Sadetzki S, Oberman B, Mandelzweig L, Chetrit A, Ben-Tal T, Jarus-Hakak A, Duvdevani S, Cardis E, Wolf M. 2008 May 1; 112(9): 1974-82.

PMID: 18361448

4. Simian Virus 40 Sequences and Expression of the Viral Large T Antigen Oncoprotein in Human Pleomorphic Adenomas of Parotid Glands. Martinelli, M, Martini, F, Rinaldi, E, Caramanico, L, Magri, E, Grandi, E, Carinci F, Pastore A, Tognon, M. 2002 Oct; 161(4): 1127-1133. PMID: PMC1867276

5. Conserved mechanism of PLAG1 activation in salivary gland tumors with and without chromosome 8q12 abnormalities: identification of SIl as a new fusion partner gene. Astrom AK, Voz ML, Kas K, Roijer E, Wedell B, Mandahl N, Van de Ven W, Mark J, Stenman G. 1999 Feb 15; 59(4): 918-923. PMID 10029085

6. Lodish H, Berk A, Zipursky SL, Matsudaira $P$, Baltimore D, Darnell
J (2000) Molecular Cell Biology.

New York: W. H. Freeman.

7.PLAG1 gene alterations in salivar y gland pleomorphic adenoma and carcinoma ex-

pleomorphicadenoma:

a combined study using chromoso me banding,

in situ hybridization and immunoc ytochemistry. Martins C, Fonseca I, Roque L, Pereira T, Ribeiro C, Bullerdiek J, Soares J. 2005 Aug; 18(8): 1048-1055. PMID:

15920557

8. Aberrant PLAG1 expression in pleomorphic adenomas of the salivary gland: a molecular genetic and immunohistochemical study. Matsuyama A, Hisaoka M, Nagao Y, Hashimoto H. 2011 May; 458(5): 583-92. PMID 21394649

9. Mucin expression in pleomorphic adenoma of salivary gland: a potential role for MUC1 as a marker to predict recurrence. Hamada T, Matsukita S, Goto M, Kitajima S, Batra S K, Irimura T, Sueyoshi K, Sugihara K, Yonezawa S. 2004; 57(8), 813-821. PMID PMC1770389 